\title{
Extending the Explanatory Scope of Evolutionary Theory: The Origin of Historical Kinds in Biology and Culture
}

Günter P. Wagner and Gary Tomlinson

Yale University

\begin{abstract}
Since its inception, evolutionary theory has experienced a number of extensions. The most important of these took the forms of the Modern Evolutionary Synthesis (MES), embracing genetics and population biology in the early $20^{\text {th }}$ century, and the Extended Evolutionary Synthesis (EES) of the last thirty years, embracing, among other factors, non-genetic forms of inheritance. While we appreciate the motivation for this recent extension, we argue that it does not go far enough, since it restricts itself to widening explanations of adaptation by adding mechanisms of inheritance and variation. A more thoroughgoing extension is needed, one that widens the explanatory scope of evolutionary theory. In addition to adaptation and its various mechanisms, evolutionary theory must recognize as a distinct intellectual challenge the origin of what we call "historical kinds." Under historical kinds we include any process that acquires a quasi-independent and traceable lineage-history in biological and cultural evolution. We develop the notion of a historical kind in a series of paradigmatic exemplars, from genes and homologues to rituals and music, and we propose a preliminary characterization.
\end{abstract}

Keywords: historical individuals, extended evolutionary synthesis, evolutionary innovation, culture

\section{Introduction}

For the last several decades, a set of concerns has exerted increasing pressure on the modern evolutionary synthesis (MES) of the mid-twentieth century, with the goal of widening the explanatory powers of evolutionary theory. The most prominent version of this expanded view now travels under the designation "extended evolutionary synthesis" (Pigliucci 2007, Pigliucci and Müller 2010, Laland, Uller et al. 2015)(http://extendedevolutionarysynthesis.com), distinguishing it from the earlier formation; the name, as its advocates usually affirm, signals its building on, rather than repudiating, the MES. The extensions built into the EES are several, as a recent summary explains (Laland, Uller et al. 2015). Where the MES starts from a genecentered perspective and emphasizes genetic inheritance, the EES balances this genetic focus with emphasis of the organism and advances an "inclusive" view of inheritance through several channels: epigenetic, physiological, ecological, social, and cultural, in addition to genetic. For proponents of the MES, the centrality of genetic inheritance suggests that evolutionary change will move in small increments, driven by natural selection and genetic drift acting upon random mutations and a few other chance mechanisms. Proponents of the EES, instead, take account of other, non-random variational mechanisms in addition to genetic change, especially phenotypic plasticity biasing development in certain directions; these suggest that evolution may not only take place incrementally but also involve saltation and threshold points. Finally, proponents of the MES maintain that natural selection, modeled as genetic variants differentially selected, 
offers an adequate explanatory model for adaptation; the EES aims to track more circuitous pathways, with organisms changing their environments ("constructing" their "niches") even as they evolve in them through selection-altering, in effect, their own fitness landscapes as well as those of the organisms around them.

This last aspect spotlights the back-and-forth relation between changeable organisms and changeable environments. It brings to the heart of evolutionary thinking "reciprocal causation" (Laland, Uller et al. 2015) - that is to say, feedback loops of mutual influence and effect among biological systems or between biological systems and their non-living surroundings. This emphasis of the role of feedback or non-linear causation is, in our view, a most important shift urged by proponents of the EES, and we will see that feedback mechanisms are active at all levels of biological and biocultural organization and change. Like the other aspects of the EES, however, feedback is not a sheer novelty in regard to the MES, but an extension of it. Its role in evolutionary thinking can be traced all the way back to Darwin's reliance on Malthusian population dynamics and the tangled bank metaphor in The Origin of Species (Darwin 1859), but it is also fair to say that this perspective did not play a major role in the research program of the MES.

Despite these moves implemented by the proponents of the EES, it remains for us too modest an expansion of evolutionary theory. We will see, for example, that an emphasis on feedback is not sufficient to describe fully the implications of reciprocal causation; we need in addition to theorize the emergence from this feedback of control mechanisms of various sorts, which come to channel or direct the feedback cycles; such controls are discussed in this final sections of this paper. More generally, the aim of the EES, as envisaged in the programmatic writing of Laland and his colleagues (Laland, Uller et al. 2015), follows closely that of the MES in one major aspect: in each case the aim is to explain adaptations, i.e., the fit of organisms to their environments. The focus on adaptation in the EES can even be seen to represent a step back in comparison with the MES, since one of the main intellectual achievements of that earlier synthesis was the recognition that the origin of species and the evolution of adaptations are two fundamentally different phenomena, requiring different explanatory models (Gould 1982). In realizing this, the architects of the MES overcame a major flaw in the Darwinian heritage; it was because of the conflation of adaptation and speciation that the foundational document of evolutionary thought, The Origin of Species, could not deliver what its title promised to explain.

This requires a bit of preliminary explanation, though we will return below to the question of speciation in greater detail. Species, as they are understood from the populationgenetic, MES point of view, are either populations reproductively isolated from other cognate populations (Mayr 1942, Mayr 1982, Mayr 1987), or populations of demographically replaceable individuals that form a lineage of descent independent from other related lineages (Templeton 1989). Lineage isolation (and speciation) can result from a number of different causes, including the chance accumulation of genetic incompatibilities in geographically isolated populations, hybridization, polyploidization, and others. Natural selection, the main explanation for adaptation, can be part of the process of speciation, but it is neither necessary nor sufficient for it. Adaptation, the modification of organismic traits by natural selection, and speciation, the origin of a novel quasi-independent lineage of descent, are different processes. 
We emphasize here the distinction between adaptation and speciation because it points to a larger problem of origination: the evolutionary and historical origination of individuals that we call historical kinds. Solving this broader problem was not an aim of the MES, and it has not featured in the EES either. But just as speciation cannot be reduced to adaptation, so this problem of the origin of kinds is a theoretical matter that falls outside the purview of adaptive evolution and natural selection. We argue for an expanded scope of evolutionary theory that will model and explain the origination of the fundamental historical kinds that occur in biology and culture and make up much of the complexity of the living and cultural worlds. We urge not only an expansion of our explanatory models - a direction we share with advocates of the EESbut also the delineation of a new area of evolutionary studies reaching beyond questions of adaptation and speciation. Achieving this will require a change in the type of questions asked both in evolutionary biology all told and also in the evolution of hominin (and other animal) cultures.

At the core of our conception of historical kinds is the idea of evolutionary individuation. The existence of evolutionary individuals has, it is clear, been recognized in evolutionary biology. A prominent example in recent evolutionary theory is Godfrey-Smith's "Darwinian individual," any member of a population that can undergo evolution through natural selection (Godfrey-Smith 2009). Darwinian individuals thus conceived exemplify the emphasis in both the MES and the EES on adaptation and population-level thinking. Other examples of individuals recognized in evolutionary biology are species and individualized parts of the body such as characters or homologs. Speciation is a particular variety of individuation. A population becomes a species if and when it undergoes a change that allows it to pursue its evolutionary history quasi-independently of other related populations. That is to say, a population becomes a species when it individuates from other populations. Such individuation is always a relational property rather than an intrinsic one; a species is individuated with respect to other populations, not because of some intrinsic essence (Ghiselin 1974, Ghiselin 1997).

What has not been so clearly recognized by evolutionists is the fact that individuation has mechanistic implications. For something to be an evolutionary and historical kind and, as such, individuated relative to other such kinds, there must be mechanisms in place that endow it with the ability to plot its quasi-independent evolutionary history. This way of thinking about individuation, we argue, applies not merely to species, but to all biological and biocultural kinds: genes, cell types, the multicellular building blocks of the body (tissues and organs), animal societies, and aspects of cultures such as distinct technologies, ritual, language, and music. Moreover, the general structures of the mechanisms by which the individuation of all these things takes place show extensive and deep similarities. These similarities afford the basis for a general theory of the individuation of biological and biocultural kinds.

This theory is a theory of process, not of fixed ontological entities. This is what we mean to signal with the notion of quasi-independence: the independence of different kinds is always only a stage in a historical process. Independence, which can seem absolute at one temporal or ontological level, will be seen, from a broader vantage, to be a product of ongoing processual change. This is as true of biological kinds as it is of cultural kinds.

Before we proceed to develop our argument, we want to offer a few words about the root of our collaboration, which, after all, is a somewhat extraordinary one between an evolutionary biologist and a musicologist. Over the last several years, we have independently 
proposed models of evolutionary and historical origination. GPW published a book on the nature of morphological characters and their origin (Wagner 2014), and GT published two books on the origin of music and of human culture in general (Tomlinson 2015, Tomlinson 2018). It was our mutual recognition of the affinities between the mechanisms we had described that motivated our collaboration.

We describe below origination events of several types in biology and culture; then we conclude by discussing the general implications of their natures and affinities.

\section{Examples of evolutionary kinds}

Genes, functional systems, and selected lineages

The gene is one of the three fundamental ontological units of biology, alongside the cell and the species. Genes are a repository, though not the only one, of biological information accumulated over evolutionary history. There are two complementary ways to introduce the gene concept, each reflecting a part of the history of our understanding.

In the Mendelian concept, genes were thought of as atomic or discrete units of inheritance, parts of the "germ plasma" that 1) cause a specific phenotypic difference (e.g., yellow or green coloration of peas) and 2) are transmitted independently from other such pieces of heritable material and unchanged (except when mutations occur). This Mendelian concept bears an obvious similarity to other $19^{\text {th }}$-century atomic concepts. It only makes sense, however, in species with sexual reproduction and recombination of their genetic material. Organisms without regular recombination, for instance bacteria, do not have "genes," at least not in the Mendelian sense of the concept.

Such organisms can, however, be considered to have genes according to the gene concept of contemporary molecular biology. In this modern concept the gene is no longer a simple atomic unit of inheritance but instead a complex functional system, with different parts ensuring the expression of the gene at the right time and place and allowing for different outputs in response to different inputs. For example, a gene that codes for a protein, called a "protein coding gene," consists of a nucleotide sequence that can be translated into an amino acid sequence as well as sequence elements necessary to initiate its transcription onto an RNA molecule and elements terminating RNA transcription. A coding gene, then, is not a discrete material element, but a segment of a continuous DNA molecule that is distinguished by a number of other functional elements such that the segment is "used by the cell" to produce a certain protein. Mutations to this DNA segment are either neutral, i.e., functionally inconsequential, or they affect the production of the protein, thus affecting also those aspects of the organism that rely on the protein's function. As a consequence, in transmission between generations, the gene appears both as a unit of inheritance and a unit of function; but this dual appearance is an emergent property, not based on a structurally unitary entity.

These two views of the gene, the transmission/genetic and the functional, are merged and connected to evolutionary theory in the gene concept employed in molecular evolution, the study of the evolutionary history of genes and genomes (Grauer and Li 2000). In molecular evolution, a gene is viewed as a segment of DNA that forms a lineage of descent, the history of 
which can be traced by analyzing, across species and populations, the similarities of the nucleotide sequence of the segment. The inheritance of the DNA sequence characteristic of a gene is caused by the direct replication of DNA, in the so-called "semi-conservative" mode of replication, meaning that each daughter copy of a gene consists of one stand of the ancestral DNA and one that was synthesized anew. Such replication leaves two copies of the original gene, identical except for rare mutations. Different variants of the same gene, or alleles, compete with each other for representation in the next generation, because they are represented by a limited number of copies in each organism in the population (usually only one or two copies). This is not the case with respect to other genes in the same genome: different genes (as opposed to different alleles) do not compete, since their representation in the next generation of organisms does not depend on their being favorably selected over one another. They are guaranteed a seat at the table, so to speak.

In a genome, the inheritance-with-competition among the alleles and the absence of competition between different genes together form the basis for the quasi-independent evolutionary history of each gene; each forms a lineage across this history. "Competition" here means that variants replace each other over time in the population, rather than persisting in parallel. Replacement can occur between functionally equivalent variants, in a process called "neutral evolution" or "genetic drift," while natural selection involves replacement on the basis of functional differences between the alleles. It is evident, then, that the allele lineages created in natural selection depend not merely on replicated segments of DNA, but in each case on the whole functional unit of which the DNA segment is a part-in other words, the whole gene, according to the concept as used in molecular biology.

Of course, there can be more complicated situations that are not captured in this simple model, for instance the possibility of stable polymorphisms, where two alleles are maintained by natural selection in the population because the heterozygote genotype is more fit than either of the homozygote genotypes. In such cases, each allele has its own evolutionary history for as long as the stable polymorphism is maintained. Stable polymorphisms can be quite old, as the famous polymorphism at the alcohol dehydrogenase locus in Drosophila melanogaster demonstrates (Hartl and Clark 1989). But these complications do not change the fundamental structure of this gene concept in molecular evolution.

An examination of genes in different genomes reveals that some genes can be more closely related to each other than to other genes in the same genome; instances are the different kinds of hemoglobin genes, or the different Hox genes, i.e., transcription factor genes that control parts of bilaterian animal development (Gehring 1998). For instance, the DNA sequences of different Hox genes are more similar to each other than each of them is to other genes in the same genome. These similarities point to their evolutionary relatedness, suggesting that the different Hox genes originated by some form of copying mechanism, which left multiple copies of one ancestral gene in different parts of the genome. Note that these copies, unlike the allelic copies discussed above, are truly different genes, which do not at present compete with each other because they are located in different parts of the genome.

The fact that different genes can be more closely related to each other than to other genes in the genome points to evolutionary events in which a gene was duplicated and each copy subsequently took on its own, quasi-independent evolutionary history or lineage of descent. Thus individuation of the sort discussed above can occur at the genetic level. Genes 
can show the pattern of evolutionary change that we argue is characteristic for all historical kinds, namely one in which evolutionary modification along a line of descent results, at rare moments, in new lines of descent, i.e., the origination of new historical kinds. In the case of genes, the origination of a new line of descent comes about through gene duplication or gene fusion. The evolutionary history of the new genes thus created, their loss or maintenance, is complicated and cannot be adequately dealt with here. Nevertheless, in their origination and modification they evince the two fundamental evolutionary patterns one finds with respect to all evolutionary, historical kinds.

The story of gene duplication, mutation, and natural selection outlined above omits an aspect of the biology that explains the functional and historical cohesion of what we think of as genes. Insertions and deletions of small fragments of DNA are frequent occurrences in evolution, and thus the expectation is that any DNA segment might diffuse into the genome and eventually become untraceable, as is happening with large chunks of non-protein coding DNA. In the case of functionally relevant parts of the genome, however, the functional integration among the parts of a gene creates an emergent mechanism that prevents the loss of identity over evolutionary time - that stabilizes, that is, the quasi-independence of the gene. While insertions and deletions still happen, they are filtered through natural selection and thus limited to those that are functionally acceptable. Hence the functional integration of the components of the gene ensures its historical/evolutionary cohesion.

\section{Historical kinds in the evolution of culture}

Cultural kinds, like biological kinds, are historical entities that emerge through evolutionary processes as units of function, forming quasi-independent lineages of these units. Discerning cultural kinds is a powerful strategy for understanding biocultural evolution. In this and two more cultural sections below we will show why this is so.

All animal cultures are characterized by fundamental processes that give rise to them and capacities that underlie these processes. Two basic processes define culture: learning and transmission (Boyd and Richerson 2005, Richerson and Boyd 2005). Culture is the process in which an organism learns such things as activities, concepts, and modes of expression in its life experience and transmits this learning to other conspecifics, especially those in the succeeding generation. Such an inclusive definition is necessary in order to take in both the deep history of culture among hominins and the cultures of some non-human animals in the world today (for example, some songbirds, cetaceans, monkeys, and apes). The fundamental capacities that underlie this dual process include attention (the ability to focus on specific stimuli from the external world while ignoring others), some considerable store of episodic and semantic memory, and at least a rudimentary shared intentionality or "theory of mind" (Carruthers and Smith 1996, Tomasello, Carpenter et al. 2005) (Knudsen 2007, Bloch 2013, Haladjian and Montemayor 2015). These in turn, in most models of cultural origins, are posited to give rise to joint attention, complexities of social interaction ("social intelligence"), and imitative behaviors (Tomasello 1999, Boyd and Richerson 2005, Sterelny 2012).

Repeated cultural gestures, formations, activities, and so forth form lineages of transmission across generations. What are transmitted in these lineages are not unitary or atomic bits of culture, but systems defined by the functions arising from the networked linkages 
of their elements, and it is these systems that form cultural kinds. These, then, resemble genes as conceived in modern molecular biology, not the atomic material units of the older Mendelian conception. Assimilating culture to the Mendelian view has been a common strategy since Richard Dawkins introduced the term "meme" to denote a discrete bit of culture (Dawkins 1976). This analogy of the Mendelian gene to elements of culture reflects, however, not merely an outmoded understanding of the gene but an inadequate understanding of cultural development and change, and its shortcomings have been repeatedly diagnosed (Sperber 1996, Deacon 1999, Bloch 2000).

The organization of cultural contents into functional systems made up of components operating in reciprocal interaction is basic to culture, just as it is basic to genes. The replication of cultural systems through generations is not, however, "semi-conservative" in the manner of genetic replication - not, at least, in the cultures of Homo sapiens over many tens of thousands of years. Because the content of culture is not a question of molecular identities such as nucleotide sequences but instead is in some degree ideational-the product of complex neural networks in interaction with sensory input - this content tends to vary incessantly rather than being preserved faithfully. These variations, however, are not so large as to make unrecognizable long continuities in lineages of cultural content and function. (Rudimentary cultures of non-human animals, and probably of now-extinct hominins, tend to display less local variation and more faithful intergenerational replication than more complex cultures. Birdsong cultures exemplify this, and it is probably also reflected, among hominins, in the famously longlived Acheulean lithic technology, stably passed on among hominin species for more than a million years (Wynn 2002, Klein 2009).

In all these features - the formation of lineages, their recognizable persistence across long timespans, and their structuring as functional systems with components linked in reciprocal interaction - certain broad manifestations of animal cultures resemble the basic units of biology (genes, cell types, and species). It makes good sense to think of such manifestations as historical kinds in part because of these resemblances. It makes good sense additionally because these cultural kinds are, like those basic biological units, direct outgrowths of evolutionary processes.

Given the transmission of learned materials that is basic to all culture, what categories of cultural manifestations will be most usefully thought of as historical kinds? All animal culture is an outgrowth of more basic and widespread animal sociality, which is a supra-individual phenomenon and necessarily plays itself out in relation to the environments inhabited by social species. Cultures, in this way, are all particular instances of social niche construction, and the emergence of historical kinds in culture starts from this environmentally situated sociality. The functional systems of cultural kinds involve such dimensions of animal behavior as communication, behaviors surrounding social events such as mating and death, providing sustenance for the group, and technology. Within these broad behavioral arenas, a cultural kind takes shape when a particular functional system coalesces and forms a lineage persisting across many generations.

The individuation of historical kinds in culture - that is, their origination and differentiation from other such kinds - comes about when elements in cultural lineages join to create a system that is novel in transforming some or all of those elements by virtue of the function of the whole system. These elements can be social patterns, ideas, materials, or niche 
interactions. The transformation that marks the individuation of the kind involves a mutual relation between them and the system, since the system could not arise without the components, while they could not be transformed without the systemic function. The nature of the components is changed in the system even as they make possible its function.

An example of such individuation is bead making, an activity widespread among Middle Paleolithic Homo sapiens reaching back more than 100,000 years (Vanhaeren and d'Errico 2006) (d'Errico and Vanhaeren 2007, White 2007). Beads - materials drilled through for hanging on straps, hair, or clothing-were produced at the cost of considerable effort, presumably because the ornaments they structured served as markers of social difference of one sort or another, in societies whose growing complexity made such marking advantageous. Beads reflect the formation of a cultural system out of several components: materials (shells, teeth, ivory, etc. for the beads; straps or clothing to hang them on); technologies and tools (drilling with awls or burins); and complex social or kinship forms differentiating ranks and statuses. Some of the components are material, while others are not. All the components, even as they construct the system, are transformed by its function: shells or teeth, once only waste-products of food harvesting, can now also be insignias of social position; a drilling technology is turned to a new use to produce these insignias; and social status itself is now made visible in material form through new kinds of manufacture and effort.

Competition among historical kinds in culture can be seen, across the evolutionary timespans through which they persist, as the biocultural selection of these kinds. The idea of cultural selection is not new, of course, but has been analyzed often since the 1980s. These analyses have used the recursion equations of the MES to model the changing frequencies of isolated cultural traits, in effect treating units of culture as if they were genetic alleles (CavalliSforza and Feldman 1981, Boyd and Richerson 2005). Such analyses reduce culture to atomic, Mendelian bits; they are ill-equipped to capture the interlinked specificities of content involved in any cultural system - even in, in our simple example, a system such as bead making. The building of cultural content into the robust functional systems that come to be historical kinds slips through their grasp. Such functional systems make a slightly larger appearance in the modeling of cultural niche construction in the EES, but even here the methodological limitations of recursion-equation modeling are felt (Odling-Smee, Laland et al. 2003, Laland, Uller et al. 2015), Tomlinson and Wagner, forthcoming).

Recognizing these limitations does not repudiate the findings of the niche constructionists or the cultural selectionists, but instead provides another instance where further extension of our models is called for. Niche construction will be crucial in any modeling of historical kinds of the cultural sort, since all culture, no matter how ideational in nature, manifests itself in the niches of the animals concerned-in, that is, their social and material lifeways in one environment or another. Feedback loops extending between populations and their environments are involved, and such feedback is basic to the dynamic of niche construction.

Nevertheless, by including the function and contents of cultural systems in this picture, we complicate and finally revise the simpler model of niche construction analyzed by EES advocates. We add to this model a systemic dimension redefining the evolutionary advantage of cultural patterns and channeling their further evolution, and we analyze specific mechanisms that work together to fix such systemic functioning in a local population. In our example of 
bead making above, conventional niche construction theory might posit advantages accruing to groups able to muster more complex social structure than other groups, as well as the use of material signs of rank in organizing such complexity. But it cannot take account of the emergence of a historical kind through which the potency of the marking of social difference is itself altered and its dispersion channeled in further cultural evolution.

\section{Species: their nature and origin}

The origin of species was briefly discussed in the introduction, but we return to speciation here because it exemplifies the problematic explanatory strategies that evolutionary biologists have employed when faced with questions of origination. The long history of research and debate about the nature and origin of species aids us in clarifying the discussion of the nature and origination of other historical kinds.

The species concept reflects the fact that living beings overwhelmingly come in distinct clusters of similarity: oak trees and blue whales come with very few intermediate forms. One needs to have quite specialized knowledge and invest considerable time and effort to find examples where this is not the case, i.e. species that, to the human eye, look very similar, socalled "sister species" (Mayr 1963). On the other hand, it is the case that there are species that consist of phenotypically distinct subpopulations but nevertheless fulfill the criteria of belonging to a single species (see below). Examples of such "polymorphic" species include honey bees, Apis mellifera, and Homo sapiens. These qualifications notwithstanding, the nature of species is that they form units of evolutionary change and for that reason are genetically and phenotypically distinct. Species are evolutionary individuals with their own beginning, history, and, eventually, end (Ghiselin 1974, Ghiselin 1997). Their specific histories unfold largely independently from one another. As a consequence, we must distinguish two modes of evolutionary change, modification within the lineage of descent, and speciation, i.e., species origination by lineage splitting or hybridization.

It is still fashionable to claim that the species concept is not well understood, or even that species is a meaningless concept. We disagree with both of these propositions. Usually they arise in cases where the criteria of species status are partially violated, probably because we are catching a set of populations in the act of forming a new pair of species and therefore find a situation in between a single species and two individuated species.

The huge variety of empirical situations we witness in our snapshots of evolutionary history has led also to a proliferation of "species concepts," which is, in our view, unwarranted. An analogy may help to explain why. For our "normal" meso-cosmic world we have a clearly defined concept of temperature, namely something related to the average energy of the particles in a material system (Chang 2004). Nevertheless, there are many ways to measure temperature, for instance by measuring the expansion of a fluid or solid with rising temperature, the wave length of the electromagnetic radiation emitted from hot bodies, or the changes in the resistance of an electric conductor, among others. All these methods measure the same phenomenon, but their variety does not necessitate different concepts of temperature itself. The same is true for the proliferation of species concepts. We may designate species by analyzing population-genetic data or by looking at the biogeography of diverse populations and analyzing the phylogenetic relationships among their mitochondrial 
DNAs, but the difference in these approaches does not require or justify different species concepts. All such methods are based on the shared recognition that some populations evolve quasi-independently of other populations, and these are what we call "species."

Another objection to the unity of the species concept is that species can arise through different mechanisms, as outlined in the introduction above. According to this objection, things that arise by different mechanisms cannot represent tokens of the same concept. We would need to conclude from such an argument that exemplars of the same kind must be produced by the same mechanisms. This, however, is an extraneous imposition on the species concept, given that species are understood through the role they play in the evolutionary process, i.e. by being a population-level unit of evolutionary change, not through the causes of their individuation. Different proximate mechanisms - for example, hybridization, geographic isolation, or ecological specialization - can lead to this individuation, but, notwithstanding these differences, they constitute tokens of the same kind. We believe that this is a general feature of historical kinds, namely that they are what they are because of the role they play in evolutionary/historical processes, not because of how they took on a form that could assume that role.

Once it has attained quasi-independence, the historical coherence of a species is maintained through processes similar to those that maintain the historical coherence of genes and cultural kinds, namely the operation of functional integration. In the case of sexually reproducing species, the integration results from the necessity that haploid genomes from the two parents, united during fertilization into a single cell, the zygote, need to be functionally compatible. When two species have separated, the genomes of each become epistatically separated. That is, due to distinct gene interactions in each species, genes that are beneficial in one species lead to deleterious interactions with the genes in the other species. Eventually this prevents genetic variants arising in one species from being accommodated in the other species. An impressive set of examples of this "epistatic exclusion" was provided some years ago by Sergey Kondraschov, who showed that alleles that are disease-causing in humans are often wild-type alleles in other mammalian species (Kondrashov, Sunyaev et al. 2002). While there are well-documented exceptions to epistatic exclusion, the bottom line remains that genetic species separation, to the degree it exists, is due to genetic incompatibilities.

\section{Coherence and autonomy in cultural systems: ritual}

Parallels between the persistence of species and the persistence of cultural systems become apparent in the wake of this discussion. Like species, historical kinds in culture are units of evolutionary change (in this case biocultural evolutionary change), and they lead to quasiindependent histories. They take on these features by virtue of their systemic structure-the interlinking of components that enable them to assume certain functional roles within a culture (as in our example of bead making systems). From this structure follow other similarities to species. As with the stability of species, so with that of cultural systems: selection for the function fulfilled by the system as a whole is paramount in bringing about stable persistence. This means that the components of a cultural kind, like the components of a genetic system characteristic of a species, must cohere in order for the kind to persist in recognizable form; 
they must maintain the networked interrelations that enable those components to function in the ways they have come to function in their selective histories.

It is the coherence of the systemic networks of a cultural historical kind, then, that renders it recognizable across evolutionary history. Such coherence is a general feature of historical kinds in culture and marks them off from the cultural contexts in which they occur, giving them varying degrees of autonomy. This is clear in the case of bead making: the shell-asinsignia occupies a place in a bead-making culture that is not only determined by its relations to the other elements of the system, but is also set apart from the shell-as-byproduct of food gathering. A similar autonomy is clear also in many different cultural kinds involving communication in non-human cultures. The "songs" of songbirds, for example, are complex patterns of vocalized sounds whose role in territorial marking and mate attraction is at least generally clear. As a biocultural system making up a cultural kind, these songs comprise a parent-to-offspring pedagogy, a sonic medium, a distinctive combinatorial structure in their design, and a dedicated biological substrate in the birds' bodies and brains that enables their production and cognitive processing (Marler and Slabbekoorn 2004). These join together to make birdsong autonomous and distinct from other bird behaviors, and this systemic assemblage enables the songs to fulfill their evolved roles.

Such autonomy is evident in the case of ritual in hominin societies, and we should think of ritual as a class of historical kinds that is pervasive in human culture. A ritual comes about through the formation of a system of gestures and/or expressions that sets itself apart from the cultural expressions and gestures around it; systemic autonomy is a constitutive feature of ritual. Thus, a puberty rite, a wedding, or a Mass asserts its autonomy in fulfilling its functionor, better, it fulfills its function by asserting its autonomy. But rituals also call for something more, another defining trait of this class of kinds as a whole. In every ritual, the internal system of gestures and expressions that makes it up must be repeatable and, moreover, repeatable in specific, socially determined moments. Rituals involve a double systematization, internal and external, and the external systematization, fixing in particular circumstances the iteration of the internal system, is as basic to the class as the internal one. Rituals are distinguished from other cultural kinds by this coordination of their (systemic) performance with events (systematically) deemed important by a society.

The individuation of historical kinds within the broad class of ritual depends on both sides of this dual systematization. The formation of a functional system of gestures and expressions and the transformation of the elements in this system by virtue of their mutual interactions are basic to all cultural kinds, including rituals; but the individuation of ritual kinds in particular requires in addition the emergence of the external systematization in which the performance comes to be predictably called for and repeated. The circumstances that evoke the full, dual systematization of ritual in modern human societies, and that must have evoked it also among our ancestors, are often life-cycle events deemed to be especially consequential in a society. They include today the marking of kinship and affiliation, of rites of passage into maturity, of pair-bonding, of death, of successful communal provisioning, of shared belief, and the like.

Most of these rituals are very ancient, and it is likely that the emergence of ritual predated Homo sapiens in deep hominin history. Evidence for this is the complexity of social coordination that archaeologists have reconstructed among several earlier hominin species, 
which rose to a level that likely involved at least nascent versions of ritual's dual systematization (Gamble 1999, Pope and Roberts 2005). The emergence of rituals and their individuation, in other words, were probably early byproducts of the general increase in the complexity of social arrangements in hominin groups. This pre-sapient emergence makes it also likely that ritual kinds took shape before the language and music characteristic of sapient culture assumed their modern forms. Ritual without modern language may seem a puzzling concept, but to see this likelihood we need only picture the complexities attested in the archaeological record among hominins as far back as half a million years ago, which involved pedagogies of tool making, divisions of labor in organized hunting or scavenging, communal sharing of the spoils that resulted, and other similar behavioral patterns. The absence in such societies of modern language need not have blocked the coalescing of ritual, because they possessed well-developed vocal and bodily communicative resources referred to today as "protolanguage" or "protodiscourse." Long before the syntax and lexicon of modern languages appeared, hominin ritual could have relied on these communicative means. We return to protodiscourse below.

\section{Cell types as biological kinds}

All life depends on cellular organization, including biological entities that are not cells themselves, like viruses, which are parasites of cellular life. The most fundamental reason for this fact is that life only exists in a state of thermodynamic disequilibrium with respect to its environment, and this necessitates the maintenance of chemical gradients between the inside and outside of the cell or organism. Cells are the fundamental, i.e. atomic, unit of life, and, in our world, only originate from other such units (omnis cellula ex cellula, Virchow 1855). As the fundamental unit, cells also form the building blocks of so-called higher forms of life, such as animals and plants. As cells arrange to form multicellular units of life, they differentiate into distinct cell types, dedicated to different functions. Probably one of the first such differentiations was that between cells dedicated to somatic functions (digestion, locomotion, etc.) and those specialized for reproduction (Buss 1987). The diversification of cell types is one of the major avenues for the evolutionary increase in organismal complexity. About five to thirty cell types may exist in the anatomically most primitive free-living animal (Trichoplax adhaerens), while about 500 are recognized for humans and other mammals - a number that is most likely an underestimate.

Only in recent years has it been fully recognized that cell types form distinct, individuated lineages of descent (Arendt 2008). This means that they are historical kinds in the sense we use the term here, and indeed they show the formal properties common to biological kinds. In addition to forming lineages of descent, they undergo modification along those lineages and can originate new lineages by lineage splitting, like species during speciation, and by hybridization, in cell type fusion ((Wagner 2014), chapter 8; (Arendt, Musser et al. 2016)). This conceptual re-evaluation of cell types as phenotypic units of evolutionary change came about through the realization that cell types can be homologous across large phylogenetic distances. To say that cell types in two species are homologous is to hypothesize 1) that the most recent common ancestor of these two species also had the same cell type and 2) that there are two unbroken chains of inheritance connecting the last common ancestor to the cell 
types in the derived species. Language begins to fail us here in the face of historical continuities, since recognizing that cell types from two species are homologous in fact amounts to the recognition that they are the same cell type.

The discovery of cell-type lineages was driven in part by the recent availability of molecular signatures that allowed identification of homologous cell types even in cases where the morphological and functional phenotypes were unrecognizably different. In these cases the cells have undergone a change of function in the course of evolution, for example, a change from a photoreceptor cell to an interneuron.

From this it is clear that cell types are analogous to genes, species, and cultural types at a formal, phenomenological level, that is, they are units of evolutionary change that form lineages along which take place modifications and rare events of origination, mostly by lineage splitting (i.e. the origin of sister cell types; Arendt 2008). Within biological kinds, however, the difference between the origination of species or genes and that of cell-types arises at the proximate level, in their modes of inheritance. Genes form lineages of descent based on direct replication of the DNA molecules, in which the descendant copies of the gene have material overlap with their mother copies (Griesemer 2000). Species arise from the separation of populations, where there is a continuity of populations from the ancestral species to the two descendant species. In contrast, cell types do not show this direct form of replication, since a cell type in a parent does not give rise directly to that cell type in the offspring. Nerve cells, for example, do not directly generate nerve cells in the baby. Instead, a process of developmental mediation is required: in each generation, the specialized cell types of the offspring arise through a process of development and differentiation from undifferentiated cells arising from the zygote. In a structural sense, we can analogize this mediation to that required in a lineage of cultural kinds: the cultural kind too is not directly replicated, but needs to be learned in each new generation.

This developmental mediation leads to a number of conceptual difficulties, in particular with respect to the ontological status of cell types. For example, Peter Godfrey-Smith (personal communication) argues that cell types cannot be historical individuals analogous to species and genes, because they do not directly replicate; though it might be granted that we can represent the relationships among cell types in different species in a way similar to a phylogenetic tree, this representation is illusory. In this argument, however, phenomenon and explanation are conflated. The phenomenon is the treelike pattern of diversification found in genes, species, and also (putatively) in cell types. One model for the formation of the tree is direct replication, which can explain the homology of genes and the relatedness of species. But to say that a phenomenon is an illusion because one particular explanation for its formation does not apply is to give the explanatory model an inappropriate ontological status. Who is to say that there are not different models of explanation applicable to different instances of the same phenomenon? We encountered the same flawed argument in discussing the nature of species and speciation. There the argument was that the species concept is meaningless because different species might have originated through different mechanisms. We rejected this argument, and we do the same here. What matters for us is the fact that the tree-like pattern of evolutionary diversification of cell types shows that they behave as evolutionary individuals. How they came to do so-whether in the same way as genes and species-is irrelevant. 
Nevertheless, if cell types are evolutionary individuals-i.e., biological/historical kindswe still require an explanation as to how the individuation proper to them comes about. We need to explain how some cells are individuated from other cells in the same body with respect to their evolutionary history. If there is no direct replication and transmission of cell types from generation to generation, what then endows a cell type with the ability to have its own evolutionary history, quasi-independent from that of other cell types? The answer to this question has not been settled, but what we have learned in recent decades about the molecular biology of cell-type differentiation and the evolution of gene regulatory networks suggests a plausible model.

Different cell types usually perform different functions in multicellular organisms. To be able to do so they express a different, though partially overlapping, set of genes than other cell types do. Different genes lead to different gene products, such as enzymes and cytoskeletal proteins, and these determine the cell types' physiological and morphological phenotypes. The genes that produce the phenotype of cell types can be called "effector genes." But how do different cell types come to express these different sets of effector genes? At one level the answer lies in the diverse signals a cell receives from other cells in the embryo, which determine its ultimate fate. In many cases, however, the signals, which can be small molecules such as steroid hormones, peptides, or ions, do not directly regulate the effector genes, but instead activate a set of genes, many of them transcription factor proteins. Several slightly different models suggest that these genes form a small regulatory network, variously called kernel (Davidson and Erwin 2006), cell type identity network (Wagner 2007, Wagner 2014), or terminal selector module (Hobert 2011). The genes in the cell type identity network, once activated by the differentiation signals, then 1) regulate the expression of effector genes, and 2) suppress, directly or indirectly, the activity of alternative cell type identity networks and their cognate effector genes.

This forms a three-layer model, encompassing signals, cell type identity network, and effector genes and/or effector modules. Using this model, we can devise a scenario for the structure of the gene regulatory network that explains the evolutionary individuality of cell types. In order to do so, we need to add one additional feature, critical for what follows, to the cell type identity network model. The transcription factor proteins produced by the genes of a cell type identity network do not act singly, but form a physical complex that has been called a "core regulatory complex" or CoRC (Arendt, Musser et al. 2016). This CoRC is the actual molecular system that regulates the gene expression specific to a particular cell type. Cell differentiation signals lead to the formation of this CoRC not only through their influence on gene transcription but also by causing post-translational modifications of the transcription factor proteins, such as phosphorylation, acetylation, ubiquitination, and others. These CoRCs are evolutionarily highly conserved and rigidly associated with the evolutionary lineage of their cognate cell types (Brunet, Fischer et al. 2016).

A CoRC regulates the expression of effector genes by binding to specific cis-regulatory elements (CREs), segments of DNA often proximate to and associated with the effector gene. In other words, in a cell type endowed with its cell type-specific CoRC, any gene with an appropriate CRE will be expressed. An effector gene can, in evolution, come under the control of a CoRC through the insertion of a transposable element, which can carry a bunch of readymade transcription factor binding sites; this scenario has been demonstrated in many cases 
(Emera and Wagner 2012, Lynch, Nnamani et al. 2015). The implications of this are profound: the phenotype of a cell type can evolve through the acquisition or loss of genes regulated by a cell type-specific CoRC. The historical continuity of a cell type identity is mediated through the evolutionary continuity of its CoRC expressed in a set of cells in the body. This model amounts to a mechanistic explanation of how a cell type can maintain its identity and thus historical independence from other cell types, and at the same time evolve a more or less arbitrary phenotype. The cohesion of the cell type, over evolutionary time, can be explained by the coevolution among the transcription factors forming the CoRC.

This scenario is a model only-a schematic simplification that serves to illustrate how cell types can have historical continuity and quasi-independent trajectories of evolution and to explain their tree-like pattern of evolutionary diversification. It suggests, nevertheless, that cell types are endowed with a molecular machinery-i.e., core-regulatory complexes of transcription factor proteins - that enables differential expression of effector genes. And it suggests also that this regulatory machinery enables the cell type-specific evolution of the cell phenotype, leading to the evolutionary modification of cell types. The evolution of novel CoRCs or cell type identity networks can thus originate a novel cell type, in turn splitting the evolutionary fate of a cell population and leading to two new cell types. This mechanistic scenario offers an explanation of the diversification of cell types along trees of phylogenetic relatedness in which direct replication of cell types plays no role.

\section{Control systems and epicycles in the formation of cultural kinds}

This model of cell type identity networks suggests that a functional system can itself give rise to regulatory or control mechanisms that shape or determine its operation. Such mechanisms, spontaneously generated from within the system, can assume long-lived stability, coherence, and autonomy. The stable CoRCs, guiding the expression of effector genes and thus the differentiation of cell types, show these features. In our view, understanding such emergent control mechanisms represents an important extension of the emphasis placed on feedback networks in the EES. In systems theory, external mechanisms controlling the feedback loops of a system but not directly a part of those loops are known as feedforward elements. We can think of control mechanisms such as CoRCs as elements generated from within a functional system that, while only quasi-independent from the system that gave rise to them, come to show such feedforward dynamics.

Emergent control mechanisms of a similar sort arise in cultural systems. Here, as in CoRCs, they take on a heightened stability and autonomy, which in this case are played out across the biocultural evolutionary situations in which they are formed. In their quasiindependence from the cultural kinds that generate them, they exert feedforward, controlling effects on the feedback cycles involved in biocultural evolution; for this reason Tomlinson has called them cultural epicycles (Tomlinson 2015, Tomlinson 2018). Epicyclic mechanisms are formed especially in complex animal cultures (though they are arguably not unknown in simpler ones), and much of the dynamism of the recent biocultural evolution of hominins may be traced to their operation in the formation of new historical kinds. What are the general conditions under which they arise? 
Remember, first, that all culture is niche constructive - that is, it manifests itself in the environmentally situated sociality of the animals that create it. The literature on niche construction has treated extensively the feedback cycles between culture and biology. Usually they are viewed this way: cultural practices of a population alter the environment in advantageous ways, giving rise to altered selective pressures on the genomes of the population; selection is favored for genes enabling or fostering the practices that advantageously alter the environment, and this pushes the population as a whole toward enhanced culture-making capacities (Odling-Smee, Laland et al. 2003, Laland, Uller et al. 2015).

Epicyclic mechanisms add a new dimension to this model. By virtue of their controlsystem impact on the feedback cycles of biocultural evolution, they drive culture forward in a specific direction, not only redoubling its niche-shaping force but also biasing its outcome. They can operate in this way because of their heightened coherence, autonomy, and stability, generated from the cultural system itself in its interactions with the niche. In this dynamic we see a parallel to the system-generated regulatory networks that control the individuation and persistence of cell types. In each case, one of the results is a tightening of the coherence of the functional system, whether cultural or genetic/epigenetic; further consequences in each case can be the strengthened autonomy of the system as a whole from surrounding systems and the increased likelihood of its persistence.

This begins to describe the dynamic by which cultural epicycles give rise to new kinds, but there is another aspect that is central to it. Because culture is niche constructive, all historical kinds that are individuated in it stand in close relation to the affordances and constraints of the environments in which they operate. In reshaping the niche, cultural/historical kinds do not merely reflect passively these environmental conditions, but instead alter them in more or less dramatic ways. The epicyclic origination and individuation of a historical kind is an especially marked event in this reshaping of environmental conditions, in which factors that had not previously functioned as affordances or constraints begin to do so. Environmental factors previously insignificant in biocultural evolution take on a new, powerful significance because of the nature and function of the coalescing cultural system itself. It is as if this system were probing a biocultural search space, bumping up against new aspects of this space in its exploration. The system, then, takes on an altered identity in tandem with its qualitative alteration of external factors relevant to its niche construction. Both coalescing system and reshaped niche can then "lock" into a new, stable conformation that facilitates further biocultural evolution along certain lines rather than others. This mutual shaping of regulatory systems within culture and those between culture and its niches has parallels in the relations of constructed niches to gene regulatory systems; see (Laubichler and Renn 2015).

An example will clarify this model. It takes the form of a set of linked inferences aiming to explain the emergence among hominins of some capacities basic to modern human language and music (for fuller accounts, see (Tomlinson 2015, Tomlinson 2018)). Language and music can each be considered a single cultural kind, not a class of cultural kinds, like ritual. This is true notwithstanding the thousands of human languages in the world today and the many more that have come and gone, and the innumerable musical practices humans have devised. The grouping of all languages and musical practices into two cultural kinds is warranted by the specificity of the components that determine not merely one but all human languages, and not just a single musical practice but all musical practices. (No such specificity of components 
extends across all rituals.) These include, in language, the dual combinatorial structuring of discrete elements-phonemes into words, words into propositions-and, in music, discrete pitch processing and metric entrainment. These features and others make up internal systems that structure human language and music; in each case, the structure enables the communicative function.

Thus, language as a whole and music as a whole distinguish themselves in their particular functional systematizations from other communicative means. Indeed, it helps, in understanding language and music as individual cultural kinds, to see them in the broader perspective of the cultural communication of many animals. This is a class of cultural kinds that extends well beyond our species. It includes birdsong, the click codes of sperm whales, the songs of humpback whales, and some communicative gestures of great apes (including some vocalizations - "gesture-calls," as one anthropologist has usefully named them; (Burling 2007)). (Note that this class does not include all animal communication but cultural communication only, that is, communication using means and expressions learned and transmitted to future generations.)

But how were language and music, distinctive human historical kinds of communication, individuated in our deep history? In each case, several cultural epicycles arose that redefined environmental or ontological constraints on communication. There is space here to outline only one of these, which militated toward the forms of discrete processing that characterize both language and music (for more, see (Tomlinson 2015, Tomlinson 2018)).

As early as half a million years ago, as we remarked in the discussion of ritual, at least some hominins lived in social groups complex enough to require well-developed communicative codes. These protolanguages or protodiscourses (Bowie 2008), to judge from archaeological reconstruction and the communication of apes and monkeys in the world today, involved varieties of bodily gesture and vocalization. The communication achieved was indexical, that is, its meanings depended on relations of pointing, contiguity, or causal connection between vocal and gestural signs and their objects. This limited the range of the communication and rendered it dependent on its immediate context, a condition that still plays an fundamental role in language today and is referred to as deixis.

The development of indexical protodiscourse progressed in tandem with the sociality around it, the one nudging the other toward greater complexity as the niche-constructive advantages of such complexity came to be felt. This resulted in a pressure to increase the number and variety of the signs employed. In vocalization - to judge, again, from non-human mammal vocalizations in the world today - the indexes were at first deployed along a graded, analogue spectrum, with continuously modulated intonational shapes, amplitudes, onsets and decays, and timbral patterns. But the multiplication of such indexes brought them up against a new constraint, not relevant to simpler protodiscourse: the ontological constraint, on any graded spectrum, that increasing the number of events will decrease the distance between them. Each index, as their number multiplied, became more and more like the ones proximate to it on the spectrum. Communicative clarity was compromised as the number of signs increased.

With this new constraint in play, a cultural epicycle took shape that militated for discrete, not graded, distinctions between signs. The altered niche, the advantageous structuring of which now depended on the enlarged vocabulary of signs in the protodiscourse, 
created a general selection for capacities that enhanced the production and comprehension of these signs; but the newly active ontological constraint made discrete production and processing an important part of those capacities. The epicycle acted as a control, driving the protodiscourse toward the "solution" of discreteness, and this feature would eventually come to be fundamental in the emergence of both language and music. Discreteness of timbre and onset and decay would mark off one uttered unit from another in the vowels, phonemes, and syllables of modern language, while discreteness of pitch would set off a cascade of new capacities, leading to the relative pitch perception, octave duplication, and scales of music today.

\section{Conclusion: the general nature and implications of historical kinds}

All the examples discussed above and many others that we could not cover here share structural features that justify gathering them under a common concept, that of the historical kind. All historical kinds are embedded in processes of generational replacement. And all are maintained in these processes through mechanisms of reiteration, either by direct, material replication, or by indirect, mediated redevelopment and informational transmission, or by both. During the process of replication, a historical kind retains a level of coherence and autonomy from other, cognate historical kinds, where coherence and autonomy are two sides of the same coin, a stabilizing dynamic that helps to define the kind. Reiteration with coherence and autonomy sets into motion a process of historical change along lines of descent, which is determined through competition, among variant historical kinds, for representation in succeeding generations.

We recognize that this concept of historical kinds has a strong affinity to GodfreySmith's "Darwinian Individual" (Godfrey-Smith 2009). But we think it also shows marked differences from, and heuristic advantages over, this idea. In the first place, it is broader, because it is more liberal with respect to the kinds of processes that can be subsumed under it. Darwinian individuals are limited to entities that show some kind of direct, material replication, with DNA being the paradigmatic example. In contrast, the modes of replication that sustain lineages of historical individuals can be either directly material or mediated by control systems, teaching, and so forth. The individuation of historical kinds, moreover, is not due to the nature of the replication process so much as to the cohesion brought about by functional integration. Most of all, our model of historical kinds points more clearly than Godfrey-Smith's Darwinian individuals to a set of processes beyond conventional models of natural selection that have still not received their due in evolutionary theory.

New historical kinds can arise through a variety of processes that establish a novel lineage of descent. Such individuation, establishing a novel historical kind, is subserved by mechanisms that enable quasi-independent change within a kind. This is the process of origination. We maintain that, while a limited set of origination events, foremost among them speciation and gene duplication, have received intense attention in evolutionary biology, the utility of the concept of origination reaches far beyond these. It can help to organize research into the origin and evolution of cell types, morphological characters, behaviors, and patterns of cultural change and variation. Even rituals, music, and bead making can fruitfully be 
conceptualized as historical kinds individuated from other cultural activities and assuming a "life and history of their own," partially decoupled from those of other cultural activities or lineages.

This idea of historical kinds also generalizes a conceptual distinction that, to our knowledge, was first fully understood in the context of species evolution and origination, namely the distinction between so-called "anagenetic" evolution, modifications that occur along a lineage of descent, and "cladogenetic" evolution, which leads to new lineages and new species. For all sorts of historical kinds we must distinguish two modes of historical change, the modification of a kind during its history, and the origination of novel kinds. These two modes of evolutionary/historical change require different research approaches stimulated by different sets of questions. In the case of the evolutionary modification of a historical kind we ask what drives historical/evolutionary change, what determines the direction of change, and how the variation and variety that feeds historical change is generated. In the case of the origination of historical kinds, instead, we ask how the new historical kind acquires its functional independence-that is, how it individuates from other such kinds-and how, once its function coalesces, it maintains its coherence and autonomy over historical and evolutionary time.

The recognition that species evolution and origination are two fundamentally different kinds of evolutionary processes, calling for different forms of scientific investigations, was a basic intellectual breakthrough in evolutionary biology of the $20^{\text {th }}$ century. We think that this idea has much broader applications and can orient biological and biocultural research in radically new and productive directions. Our argument engages with a recent neoWhiteheadian move in the philosophy of biology away from ontologies of substance toward processual ontologies (Dupre 2012, Dupre and Nicholson 2018, Griesemer 2018, Love 2018). We believe that only with a strategy such as this can we open up the full implications of the historicizing of life sciences that began to take shape in the writings of Darwin and his predecessors. And we are convinced that only with this strategy can we discern the deep processual and mechanistic commonalities that link the origination of biological kinds with that of the cultural kinds some animals create. 


\section{References:}

Arendt, D. (2008). "The evolution of cell types in animals: emerging principles from molecualr studies. ." Nature Reviews Genetics 9: 868-882.

Arendt, D., J. M. Musser, C. V. H. Baker, A. Bergman, C. Cepko, D. H. Erwin, M. Pavlicev, G. Schlosser, S. Widder, M. D. Laubichler and G. P. Wagner (2016). "The origin and evolution of cell types." Nat Rev Genet 17(12): 744-757.

Bloch, M. (2000). A Well-Disposed Social Anthropologist's Problem with Memes. Darwinizing Culture: The Status of Memetics as a Science. R. Aunger. Oxford, UK, Oxford University Press. Bloch, M. (2013). In and Out of Each Other's Bodies: Theory of Mind, Evolution, Truth, and the Nature of the Social. Boulder, CO, Paradigm.

Bowie, J. (2008). "Proto-discourse and the emergence of compositionality. ." Interaction Studies 9: 18-33.

Boyd, R. and P. J. Richerson (2005). The origin and evolution of cultures. New York, Oxford University Press.

Brunet, T., A. H. Fischer, P. R. Steinmetz, A. Lauri, P. Bertucci and D. Arendt (2016). "The evolutionary origin of bilaterian smooth and striated myocytes." Elife $\mathbf{5}$.

Burling, R. (2007). The talking ape: how language evolved. . Oxford, Oxford University Press. Buss, L. W. (1987). The Evolution of Individuality. New York, Columbia University Press. Carruthers, P. and P. K. Smith (1996). Theories of Theories of Mind. . Cambridge, UK. , Cambridge University Press.

Cavalli-Sforza, L. L. and M. W. Feldman (1981). Cultural transmission and evolution: a quantitative approach. Princeton, NJ, Princeton University Press.

Chang, H. (2004). Inventing temperature: measurement and scientfic progress. , Oxford University Press.

d'Errico, F. and M. Vanhaeren (2007). Evolution or Revolution? New Evidence for the Origin of Symbolic Behaviour In and Out of Africa. Rethinking the Human Revolution. P. Mellars, K. Boyle, O. Bar-Yosef and C. Stringer. Cambridge, The McDonald Institute: 275-286.

Darwin, C. R. (1859). The Origin of Species. London, John Murray.

Davidson, E. H. and D. H. Erwin (2006). "Gene regulatory networks and the evolution of animal body plans." Science 311: 796-800.

Dawkins, R. (1976). The selfish gene. New York, Oxford University Press.

Deacon, T. (1999). "Memes as Signs: The Trouble with Memes (and What to Do about It)."

Semiotic Rev. of Books 10: 1-3.

Dupre, J. (2012). Processes of life: essays in the philosophy of biology. . Oxford, Oxford University Press.

Dupre, J. and D. J. Nicholson (2018). A manifesto for a processural philosophy of biology. . Everything flows: towards a processural philosophy of biology. D. J. Nichnolson and J. Dupre. Oxford, Oxford University Press: 3-45.

Emera, D. and G. P. Wagner (2012). "Transformation of a transposon into a derived prolactin promoter with function during human pregnancy." Proc Natl Acad Sci U S A 109(28): 1124611251. 
Gamble, C. (1999). The Palaeolithic Societies of Europe. Cambridge, UK, Cambridge Univeristy Press.

Gehring, W. (1998). Master Control Genes in Development and Evolution: the homeobox story. New Haven, CT, Yale University Press.

Ghiselin, M. T. (1974). "A radical solution to the species problem. ." Syst Zool 23: 536-544.

Ghiselin, M. T. (1997). Metaphysics and the origin of species. New York, State University of New York Press.

Godfrey-Smith, P. (2009). Darwinian populations and natural selection. Oxford, UK, Oxford University Press.

Gould, S. J. (1982). Introduction. Genetics and the Origin of Species. T. Dobzhansky. New York, Columbia University Press: xvii-xli.

Grauer, D. and W.-H. Li (2000). Fundamentals of molecular evolution. Sunderland, MA, Sinauer Assoc. .

Griesemer, J. (2000). "Development, cultiure and the units of inheritance. ." Phil. Sciene 67: S348-S368.

Griesemer, J. (2018). Individuation of developmental systems. Individuation, process, and scientific practices. O. Bueno, R.-L. Chen and M. B. Fagan. Oxford, Oxford University Press: 137164.

Haladjian, H. H. and C. Montemayor (2015). "On the evolution of conscious attention." Psychon Bull Rev 22(3): 595-613.

Hartl, D. L. and A. G. Clark (1989). Principles of Population Genetics. Sunderland, MA, Sinauer Associates.

Hobert, O. (2011). "Regulation of terminal differentiation programs in the nervous system." Annu Rev Cell Dev Biol 27: 681-696.

Klein, R. G. (2009). The Human Career: Human Biological and Cultural Origins. Chicago, IL, University of Chicago Press.

Knudsen, E. I. (2007). "Fundamental components of attention." Annu Rev Neurosci 30: 57-78.

Kondrashov, A. S., S. Sunyaev and F. A. Kondrashov (2002). "Dobzhansky-Muller incompatibilities in protein evolution." Proc Natl Acad Sci U S A 99(23): 14878-14883. Laland, K. N., T. Uller, M. W. Feldman, K. Sterelny, G. B. Muller, A. Moczek, E. Jablonka and J. Odling-Smee (2015). "The extended evolutionary synthesis: its structure, assumptions and predictions." Proc Biol Sci 282(1813): 20151019.

Laubichler, M. D. and J. Renn (2015). "Extended evolution: A conceptual framework for integrating regulatory networks and niche construction." J Exp Zool B Mol Dev Evol 324(7): 565577.

Love, A. C. (2018). Individuation, individuality, and experimental practice in developmental biology. . Individuation, process, and scientific practices. . O. Bueno, R.-L. Chen and M. B. Fagan. Oxford, Oxford University Press: 165-191.

Lynch, V. J., M. C. Nnamani, A. Kapusta, K. Brayer, S. L. Plaza, E. C. Mazur, D. Emera, S. Z. Sheikh, F. Grutzner, S. Bauersachs, A. Graf, S. L. Young, J. D. Lieb, F. J. DeMayo, C. Feschotte and G. P. Wagner (2015). "Ancient transposable elements transformed the uterine regulatory landscape and transcriptome during the evolution of mammalian pregnancy." Cell Rep 10(4): 551-561. Mayr, E. (1942). Systematics and the Origin of Species. New York, Columbia University Press. Mayr, E. (1963). Animal species and evolution. . Cambridge, MA, Harvard University Press. 
Mayr, E. (1982). The Growth of Biological Thought. Cambridge, London, The Belknap Press. Mayr, E. (1987). "The ontological status of species: scientific progress and philosophical terminology." Biol Phil 2: 145-166.

Odling-Smee, F. J., K. N. Laland and M. W. Feldman (2003). Niche Construction: The Neglected Process in Evolution. Princeton, NJ, Princeton University Press.

Pigliucci, M. (2007). "Do we need an extended evolutionary synthesis?" Evolution 61(12): 27432749.

Pigliucci, M. and G. B. Müller, Eds. (2010). Evolution: the extended synthesis. . Cambridge, MA, MIT Press.

Pope, M. and M. Roberts (2005). Observations on the Relationship between Palaeolithic Individuals and Artefact Scatters at the Pleistocene Site of Boxgrove, UK. The Hominid Individual in Context: Archaeological Investigations of Lower and Middle Palaeolithic Landscapes, Locales, and Artefacts. C. Gamble and M. Porr. New York, Routledge: 81-97. Richerson, P. J. and R. Boyd (2005). Not by genes alone: how culture transformed human evolution. . Chicago and London, University of Chicago Press.

Sperber, D. (1996). Explaining culture : a naturalistic approach. Oxford, UK ; Cambridge, Mass., Blackwell.

Sterelny, K. (2012). The Evolved Apprentice: How Evolution Made Humans Unique. Cambridge, MA, MIT Press.

Templeton, A. (1989). The meaning of species and speciation: A genetic Perspective. Speciation and its Consequences. D. Otte and J. A. Endler. Sunderland, MA, Sinauer: 3-27.

Tomasello, M. (1999). The Cultural Origins of Human Cognition. Cambridge, MA, Harvard University Press.

Tomasello, M., M. Carpenter, J. Call, T. Behne and H. Moll (2005). "Understanding and sharing intentions: the origins of cultural cognition." Behav Brain Sci 28(5): 675-691; discussion 691735.

Tomlinson, G. (2015). A Million Years of Music: the Emergence of Human Modernity. . New York, Zone Books.

Tomlinson, G. (2018). Culture and the course of human evolution. Chicago and London, Chicago University Press.

Vanhaeren, M. and F. d'Errico (2006). "Aurignacian Ethno-linguistic Geography of Europe Revealed by Personal Ornaments." J. Archeological Sci. 33: 1105-1128.

Wagner, G. P. (2007). "The developmental genetics of homology." Nature Rev. Genetics 8: 473479.

Wagner, G. P. (2014). Homology, Genes and Evolutionary Innovation. Princeton, NJ, Princeton University Press.

White, R. (2007). Systems of Personal Ornamentation in the Early Upper Palaeoithic:

Methodological Challenges and New Observations. Rethinking the Human Revolution. P.

Mellars, K. Boyle, O. Bar-Yosef and C. Stringer. Cambridge, The McDonald Institute: 287-302.

Wynn, T. (2002). "Archaeology and cognitive evolution." Behav Brain Sci 25(3): 389-402;

discussion 403-338. 